OPEN ACCESS

Edited by:

Zuhua $\mathrm{He}$,

Chinese Academy of Sciences, China

Reviewed by:

Yonghong Wang,

Chinese Academy of Sciences, China

Lin Zhang,

Chinese Academy of Sciences, China

*Correspondence:

Maria C. Rebolledo

m.c.rebolledo@cgiar.org

Specialty section:

This article was submitted to

Plant Physiology,

a section of the journal

Frontiers in Plant Science

Received: 30 April 2016

Accepted: 30 August 2016

Published: 20 September 2016

Citation:

Rebolledo MC, Peña AL, Duitama J, Cruz DF, Dingkuhn M, Grenier $C$ and

Tohme J (2016) Combining Image

Analysis, Genome Wide Association

Studies and Different Field Trials to

Reveal Stable Genetic Regions

Related to Panicle Architecture and the Number of Spikelets per Panicle in

Rice. Front. Plant Sci. 7:1384. doi: 10.3389/fpls.2016.01384

\section{Combining Image Analysis, Genome Wide Association Studies and Different Field Trials to Reveal Stable Genetic Regions Related to Panicle Architecture and the Number of Spikelets per Panicle in Rice}

\author{
Maria C. Rebolledo ${ }^{1 *}$, Alexandra L. Peña ${ }^{1}$, Jorge Duitama ${ }^{1}$, Daniel F. Cruz ${ }^{1}$, \\ Michael Dingkuhn ${ }^{1,2}$, Cecile Grenier ${ }^{1,2}$ and Joe Tohme ${ }^{1}$
}

${ }^{1}$ Agrobiodiversity, International Center for Tropical Agriculture, Palmira, Colombia, ${ }^{2}$ Agricultural Research for Development CIRAD, Unités Mixtes de Recherche - Amélioration Génétique et Adaptation des Plantes, Montpellier, France

Number of spikelets per panicle (NSP) is a key trait to increase yield potential in rice (O. sativa). The architecture of the rice inflorescence which is mainly determined by the length and number of primary (PBL and PBN) and secondary (SBL and SBN) branches can influence NSP. Although several genes controlling panicle architecture and NSP in rice have been identified, there is little evidence of (i) the genetic control of panicle architecture and NSP in different environments and (ii) the presence of stable genetic associations with panicle architecture across environments. This study combines image phenotyping of 225 accessions belonging to a genetic diversity array of indica rice grown under irrigated field condition in two different environments and Genome Wide Association Studies (GWAS) based on the genotyping of the diversity panel, providing 83,374 SNPS. Accessions sown under direct seeding in one environement had reduced Panicle Length $(\mathrm{PL}), \mathrm{NSP}, \mathrm{PBN}, \mathrm{PBL}, \mathrm{SBN}$, and SBL compared to those established under transplanting in the second environment. Across environments, NSP was significantly and positively correlated with PBN, SBN and PBL. However, the length of branches (PBL and SBL) was not significantly correlated with variables related to number of branches (PBN and SBN), suggesting independent genetic control. Twenty- three GWAS sites were detected with $P \leq 1.0 \mathrm{E}-04$ and 27 GWAS sites with $p \leq 5.9 \mathrm{E}-04$. We found 17 GWAS sites related to NSP, 10 for PBN and 11 for SBN, 7 for PBL and 11 for SBL. This study revealed new regions related to NSP, but only three associations were related to both branching number (PBN and SBN) and NSP. Two GWAS sites associated with SBL and SBN were stable across contrasting environments and were not related to genes previously reported. The new regions reported in this study can help improving NSP in rice for both direct seeded and transplanted conditions. The integrated approach of 
high-throughput phenotyping, multi-environment field trials and GWAS has the potential to dissect complex traits, such as NSP, into less complex traits and to match single nucleotide polymorphisms with relevant function under different environments, offering a potential use for molecular breeding.

Keywords: GWAS, inflorescence architecture, yield, rice, direct seeding, image analysis

\section{INTRODUCTION}

Growing world population and the effects of global climate change are increasing the demand for higher crop yields. To meet rice demand in 2050, annual increase production has to increase from the current $1-2.4 \%$ by 2050 (Ray et al., 2013). New rice varieties with high yield potential have been a major target of crop improvement in rice (Peng et al., 1999). A global tendency has been to shift from puddled-transplanted rice production to direct seeding (DRS) of rice in irrigated systems to save labor and water resources (Kumar and Ladha, 2011). In Asia and Africa farmers are considering shifting to DRS (Farooq et al., 2011), however varieties currently used for DRS were bred for transplanted culture in puddle soils.

Under both production systems increasing sink size at flowering is a priority trait to increase yield potential in rice (Dingkuhn et al., 1991, 2015; Foulkes et al., 2011). One approach to increase sink size at flowering is to increase the number of spikletes per panicle (Peng et al., 1999). The panicle consists of a main axis called rachis with primary, secondary, and higher order branches bearing the spikelets. The number and dimensions of branches vary among cultivars (Ikeda et al., 2004), and will define the final architecture of the panicle. High number of secondary and primary branches and long primary branches were associated with high number of spikelets per panicle in rice (Miura et al., 2010; Fujita et al., 2013). Therefore, the genetic improvement of the number of spikelets per panicle may benefit from its dissection into component traits of lesser genetic complexity, such as panicle architecture traits (AL-Tam et al., 2013; Crowell et al., 2014).

The genetic control of panicle architecture plays an important role in rice production. Large variation was observed for panicle architecture traits among five different rice sub species (Crowell et al., 2014, 2016). To date, several genes and QTLs related to panicle architecture and affecting the number of spikelets per panicle (as reviewed by Sreenivasulu and Schnurbusch, 2012; Liang et al., 2014) have been reported. Genes such as Gn1a, OsSPS1, and SPIKE with a positive effect on the number of secondary branches also showed a significant effect on the number of spikelets per panicle and yield (Ashikari et al., 2005; Fujita et al., 2013; Hashida et al., 2013). Many QTLs related to the number and length of secondary branches with small but cumulative phenotypic effect on the number of spikelets per panicle were reported (Ando et al., 2008; Peng et al., 2014). In addition, the length of primary branches has been shown to be positively related to the number of spikelets per panicle ( $\mathrm{Li}$ et al., 2009). Understanding the relationship between panicle architecture traits and the number of spikelets per panicle will help to identify trait combinations related to a high number of spikletes per panicle that could be used for breeding.

A panicle is a complex structure and the characterization of the branching pattern for breeding has also been limited by the unavailability of appropriate screening tools. Recently, open access image analysis software packages were released to analyze panicle architecture (AL-Tam et al., 2013; Crowell et al., 2014), allowing a rapid description of each panicle architecture trait. The study of large diversity panels using Genome Wide Association Studies (GWAS) allows capturing the available allelic diversity on the loci of interest with a high physical map resolution due to the small linkage disequilibrium (LD). It is also an efficient way to dissect the genetic architecture of complex traits and a powerful tool for crop breeding (Norton et al., 2014; Rebolledo et al., 2015; Ueda et al., 2015). Using both methods (GWAS and image analysis), Crowell et al. (2016) revealed a large number of GWAS sites controlling panicle architecture. However, the relation with the final number of spikelets per panicle was not established, and relevant traits such as secondary branch number and length (Xu et al., 2004) were not addressed.

Identifying the genes responsible for intraspecific variation in the number of spikelets per panicle has been challenging because of the polygenic nature and the environmental sensitivity of panicle architecture traits. In fact, inconsistent detection and variable effects of QTLs controlling the number of spikelets per panicle across environments in rice (Zhuang et al., 1997; Li et al., 2012) revealed genotype by environment (GXE) interactions for this complex trait. Panicle architecture traits are also stongly influenced by management practices (Huang et al., 2011; Dixit et al., 2015), temperature (Laza et al., 2015), and drought (Pantuwan et al., 2002). Thus, the detection of QTLs depends on both the genetic background (parental combinations) and the environment where the experiments were conducted, resulting sometimes in partly non-overlapping sets of QTLs (Li et al., 2012; Wade et al., 2015). These results point to the necessity of assessing the genetic and phenotypic control of the number of spikelets per panicle and panicle branching for specific genetic backgrounds and environments. To our knowledge this has not been done for panicle architecture and the number of spikelets per panicle in rice.

Rice breeding will need tools to determine the ideal panicle architecture to increase the number of spikelets per panicle either under a specific or for a broad range of conditions. This study aims at extracting functional concepts to understand the physiological and genetic control of panicle architecture and the number of spikelets per panicle using an indica diversity panel, an image phenotyping tool and two contrasting environments to 
reveal GWAS sites that are either environement specific or stable across the environments.

\section{MATERIALS AND METHODS}

\section{Association Mapping Panel}

The population studied represented the diversity within the indica sub-species (http://ricephenonetwork.irri.org) covering improved and traditional varieties from all tropical regions. For the present study, the association mapping panel consists of a sub-sample of 225 indica accessions obtained from the International Rice Research Institute (IRRI) seedbank. Among the 225 accessions, 51 originate from Africa, 12 from South and Central America, 120 from South Asia and 42 from West and East Asia (Table S1).

\section{Phenotypic Evaluation \\ Site Description and Crop Establishment}

The experiments were conducted under irrigated conditions during the dry season (May to August) in CIAT Palmira $\left(76^{\circ} 21^{\prime} \mathrm{W}, 3^{\circ} 30^{\prime} \mathrm{N}\right.$, and $967 \mathrm{~m}$ elevation). The experiment was established under transplanting conditions (Exp1 TR) in 2013 and under direct seeding (Exp2 DRS) in 2014. For both experiments, the soil was a silt-loam soil with particle distribution of $10 \%$ clay, $80 \%$ silt, and $10 \%$ sand. The experimental design was a randomized complete block design with 3 replications and 225 plots. The 225 accessions were sown at the same time and grouped by previously estimated maturity date and plant height to facilitate measurements.

In 2013, seeds from the 225 accessions were sown in plastic trays and seedlings transplanted, 20 days after emergence, at one seedling per hill with a hill spacing of $0.2 \times 0.2 \mathrm{~m}$. Plot size was $3.0 \times 0.6 \mathrm{~m}$, for a plot density of 25 plants $/ \mathrm{m}^{2}$. Plots were maintained under lowland conditions with a water depth of $5 \mathrm{~cm}$, after transplanting until 2 weeks after flowering. Phosphorus and potassium were applied at $59.8 \mathrm{~kg} \mathrm{ha}^{-1} \mathrm{P}_{2} \mathrm{O}_{5}$ and $116.6 \mathrm{~kg} \mathrm{ha}^{-1}$ $\mathrm{K}_{2} \mathrm{O}$. A total of $207.4 \mathrm{~kg} \mathrm{ha}^{-1} \mathrm{~N}, 20.8 \mathrm{~kg} \mathrm{ha}^{-1} \mathrm{Fe}$, and $2.8 \mathrm{~kg} \mathrm{ha}^{-1}$ $\mathrm{Zn}$ was applied. The top soil contained $27.5 \mathrm{~g}$ organic $\mathrm{C} \mathrm{kg}^{-1}$ and 1.3 total $\mathrm{N} \mathrm{kg}^{-1}$ with $\mathrm{pH} 8.2$.

In 2014 , mechanical sowing was done in dry soil with a plot size of $3.0 \times 1.0 \mathrm{~m}$ and a plot density of 80 plants $/ \mathrm{m}^{2}$. Sprinkler irrigation was applied after sowing. Fertilizers used were $145.9 \mathrm{~kg}$ $\mathrm{ha}^{-1}$ of $\mathrm{N}, 40.5 \mathrm{~kg} \mathrm{ha}^{-1} \mathrm{P}_{2} \mathrm{O}_{5}, 17.6 \mathrm{~kg} \mathrm{ha}^{-1} \mathrm{Fe}$, and $2.8 \mathrm{~kg} \mathrm{ha}^{-1}$ $\mathrm{Zn}$. Twenty days after sowing the field was maintained under lowland condition with a water depth of $5 \mathrm{~cm}$, until 2 weeks after flowering. The top soil contained $20.5 \mathrm{~g}$ organic $\mathrm{C} \mathrm{kg}^{-1}$ and 1.18 total $\mathrm{N} \mathrm{kg}^{-1}$ with a $\mathrm{pH} 7.5$.

For both experiments weeds were controlled manually, and by herbicide applications at pre and post-emergence.

Weather data was collected daily, at one-meter distance from the experimental trial, and with a $30 \mathrm{~min}$ time step in a data logger connected with radiation (silicon pyranometer sensor (SLIB-M003), temperature and humidity sensors (S-THB-M002) (Onset Computer Corporation, Bourne, USA). Average solar radiation, minimum and maximum temperature, minimum and maximum humidity for each experiment are presented in supplementary table (Table S2).

\section{Measurements}

\section{Panicle architecture}

In both experiments, the main stem of two plants in each plot was tagged 30 days after sowing. At flowering tagged stems were harvested with its respective panicles. Each panicle was spread out on a white background and held in place with metal pins under a sticky paper. A total of 1350 panicles were dissected using P-TRAP software (AL-Tam et al., 2013). Panicle length (PL), number of primary (PBN) and secondary (SBN) branches, and length of primary (PBL) and secondary branches (SBL) were extracted from image analysis (Table 1, Table S3).

\section{Number of spikelets per panicle}

At harvest, panicles were sampled from central rows, within a soil base area of $0.2 \mathrm{~m}^{2}$ in Exp1-TR and of $0.125 \mathrm{~m}^{2}$ in Exp2-DRS. Filled and unfilled spikelets per panicle were separated from the rachis manually, and counted using the seed counter (Data Count S JR, Data Technologies) (Table S3).

\section{Statistical Analysis}

The descriptive analysis and analysis of variance were performed with SAS v9.3 (SAS Institute, Cary NC, USA) with PROC MEAN and PROC MIXED, respectively. ANOVA test was applied considering genotype as fixed effect and block and group as random effect. Means were adjusted for blocks and group factor. Histograms of frequency distribution were conducted with PROC SGPLOT and broad-sense heritability was computed using PROC MIXED model in SAS. Pearson correlation was performed using R v3.1.2 (www.R-project.org).

\section{Genotypic Data}

The panel included in this study is a subset of 329 indica accessions that were genotyped using the genotype by sequencing (GBS) protocol (Elshire et al., 2011) at Cornell University. Raw reads were demultiplexed and aligned to the rice reference genome (Os-Nipponbare-Reference-IRGSP-1.0) (Kawahara et al., 2013), and variants were identified using the NGSEP pipeline (Duitama et al., 2014). This procedure provided a raw catalog of 690 thousand variable sites across the genome.

From these panel, the following filters were applied to build a curated SNP dataset available for GWAS of 91,591 SNPs with $22.8 \%$ of missing data: minor allele frequency (MAF) $\geq 0.05$ and observed heterozygosity $\leq 0.01$ (Perea et al., 2016). Missing data was imputed with the implementation of the FastPhase Hidden Markov Model (Scheet and Stephens, 2006) available at NGSEP. Comparison with SNP calls from whole genome

TABLE 1 | Trait analyzed on the diversity panel of Indica rice.

\begin{tabular}{lll}
\hline Variable & Name & Measurement unit \\
\hline PBN & Primary branch number & Number/panicle \\
SBN & Secondary branch number & Number/panicle \\
PBL & Primary branch length & $\mathrm{cm} /$ panicle \\
SBL & Secondary branch length & $\mathrm{cm} /$ panicle \\
PL & Panicle length & $\mathrm{cm} /$ panicle \\
NSP & Number of spikelets per panicle & Number/panicle
\end{tabular}


sequencing (WGS) data from 27 varieties included in the 3000 rice genomes project (3KRGP, The 3000 rice genomes project, 2014) allowed to predict an estimated error rate of $0.85 \%$ for the non-imputed dataset and of $1.2 \%$ for the imputed dataset. Finally, genotype data for the 225 accessions phenotyped in this study was selected from the imputed dataset and a MAF filter $(\mathrm{MAF}>0.05)$ was executed again to avoid spurious associations due to low frequency alleles, leading to a final dataset of 83,374 SNPs.

\section{Genomic Wide Association Analysis}

Association mapping was performed using TASSEL v5.2.8, a genotypic matrix of 83,374 SNPs and a phenotypic matrix with 225 accessions. To detect associations between SNP and panicle architecture related traits a mixed linear model (MLM) was applied involving a kinship matrix as a random effect generated using the pairwise identity by state distance implemented in TASSEL v5.2.8.

Plots representing GWAS results (Manhattan and QuantileQuantile plots) were performed using the packages QQman in R 3.2.2 (www.R-project.org).

Significance threshold for association detection was set to $P<1.1 \mathrm{E}-04$. Within a $75 \mathrm{~kb}$ region (LD reported in the indica sub-specie, Mather et al., 2007) any SNPs and genes linked to the detected peak with a strong $\mathrm{LD}\left(r^{2}>0.7\right)$ were considered as a unique region and defined as a GWAS site. To identify genes potentially linked to the detected SNPs, we used the gene database Gramene, a comparative resource for plants (www.gramene.org).

\section{RESULTS}

\section{Range of Variation in the Diversity Panel for the Number of Spikelets per Panicle and Panicle Architecture under Contrasting Conditions}

We observed significant differences $(p<0.001)$ for all measured variables between both experiments (Table 2). Average NSP was 143 in Exp1-TR and 102 in Exp2-DRS and average PL was $23.98 \mathrm{~cm}$ in Exp1-TR and $22.06 \mathrm{~cm}$ in Exp2-DRS (Table 2). For panicle architecture related traits, $\mathrm{PBN}, \mathrm{SBN}$ and SBL showed higher values in Exp1-TR than in Exp2-DRS while PBL had higher values in Exp2-DRS than in Exp1-TR.

Image analysis allowed to identify panicle architecture differences within the indica diversity panel (Figure 1). The genotypic variation for NSP was large, ranging from 51 to 169 spikelets per panicle in Exp2-DRS and from 73 to 258 spikelets per panicle in Exp1-TR (Figure 1E). PL differences between varieties ranged from 13.3 to $37.9 \mathrm{~cm}$ in Exp1-TR and from 13.1 to $31.5 \mathrm{~cm}$ in Exp2-DRS. PBN values ranged from 9 to 22 in Exp1-TR and from 6 to 14 in Exp2-DRS (Figure 1A). PBL, SBL, and SBN showed a wide distribution for each experiment (Figures 1B-D), however differences between experiments were lower than those observed for PBN. Since the experiments were conducted under different sowing conditions, we computed broad sense heritability for each experiment. All the measured variables showed broad sense heritability values higher than 0.7 (Table 2).

\section{Correlations between Panicle Architecture Traits and the Number of Spikelets per \\ Panicle}

For all variables, the correlation between Exp1-TR and Exp2DRS across genotypes was positive and significant $(p<0.001)$ (Table 3). The highest correlation between experiments was observed for NSP and SBL while the lowest correlation was observed for PBL and PL (Table 3).

Correlations between variables showed similar patterns for Exp1-TR and Exp2-DRS (Table 3): (i) PL was significantly and positively correlated to all the measured panicle architecture traits (PBL, PBN, SBL, SBN) (Table 3), (ii) PBN was positively and significantly $(p<0.001)$ correlated with SBN, (iii) PBL was positively and significantly $(p<0.001)$ correlated with SBL, and (iv) PBL was significantly $(p<0.001)$ and positively correlated with SBN.

Interestingly, PBN was not significantly correlated with PBL, and SBN was not significantly correlated with SBL in any experiment. Suggesting that, considering the same rank of branching, greater branch number was not associated with greater branch length.

Secondary branches originate from primary branches therefore it was not surprising that PBL was significantly and positively correlated with SBN (Table 3).

NSP was positive and significantly $(p<0.01)$ related to both the number and length of branches (PBL, PBN, and SBN) within both Exp1-TR and Exp2-DRS; but no significant correlation was observed between NSP and SBL (Table 3). The correlation between NSP and variables related to the number of branches (PBN,SBN) was higher than the correlation with variables related to the length of branches (PBL) (Table 3). PL was not significantly correlated with NSP in Exp1-TR, while the correlation between PL and NSP in Exp2-DRS was positive and significant $(p<0.05)$. Since NSP was not significantly correlated with PL, PL was not considered for the GWAS study.

\section{GWAS for Panicle Architecture Traits and the Number of Spikelets Per Panicle}

In the final dataset of 83,374 SNPs the number of SNPs available for analysis resulted in a mean distance between neighboring SNPs was $4.7 \mathrm{kbp}$. The coverage of the indica genome could thus be considered as saturating when considering a linkage disequilibrium spanning $75 \mathrm{kbp}$ on average in this population (Mather et al., 2007). The genome wide map of SNP variation thus gave a good coverage of the genome.

We used a compressed MLM to identify association signals. The Manhattan plots and Quantile-Quantile plots of all the traits are shown in Figure 2. Twenty three GWAS sites were significantly associated with panicle architecture and NSP with a threshold of $p<1.0 \mathrm{E}-04$, and 27 with $P=5.9 \mathrm{E}-04$ (Figure 3, Table S4).

A total of 14 GWAS sites were associated with NSP, 17 GWAS sites were associated with branch length (6 to PBL and 11 to $\mathrm{SBL}$ ), and 19 GWAS sites were associated with the number of branches (11 with SBN, and 8 with PBN). NSP collocated with 
TABLE 2 | Means and broad sense heritability for each trait measured in both experiments.

\begin{tabular}{|c|c|c|c|c|c|c|}
\hline Trait & \multicolumn{3}{|c|}{ Exp1-TR } & \multicolumn{3}{|c|}{ Exp2-DRS } \\
\hline SBN & $37.73 a$ & 11.17 & 0.81 & $25.33 b$ & 7.58 & 0.79 \\
\hline PBL & $9.27 b$ & 1.24 & 0.87 & $10.28 \mathrm{a}$ & 1.4 & 0.84 \\
\hline SBL & $2.63 a$ & 0.32 & 0.9 & $2.48 b$ & 0.31 & 0.89 \\
\hline NSP & $143.11 \mathrm{a}$ & 34.32 & 0.81 & $102.57 \mathrm{~b}$ & 22.71 & 0.77 \\
\hline
\end{tabular}

Tukey test was used to calculate differences between years.

Means with different letters are significantly different at $p<0.0001$.
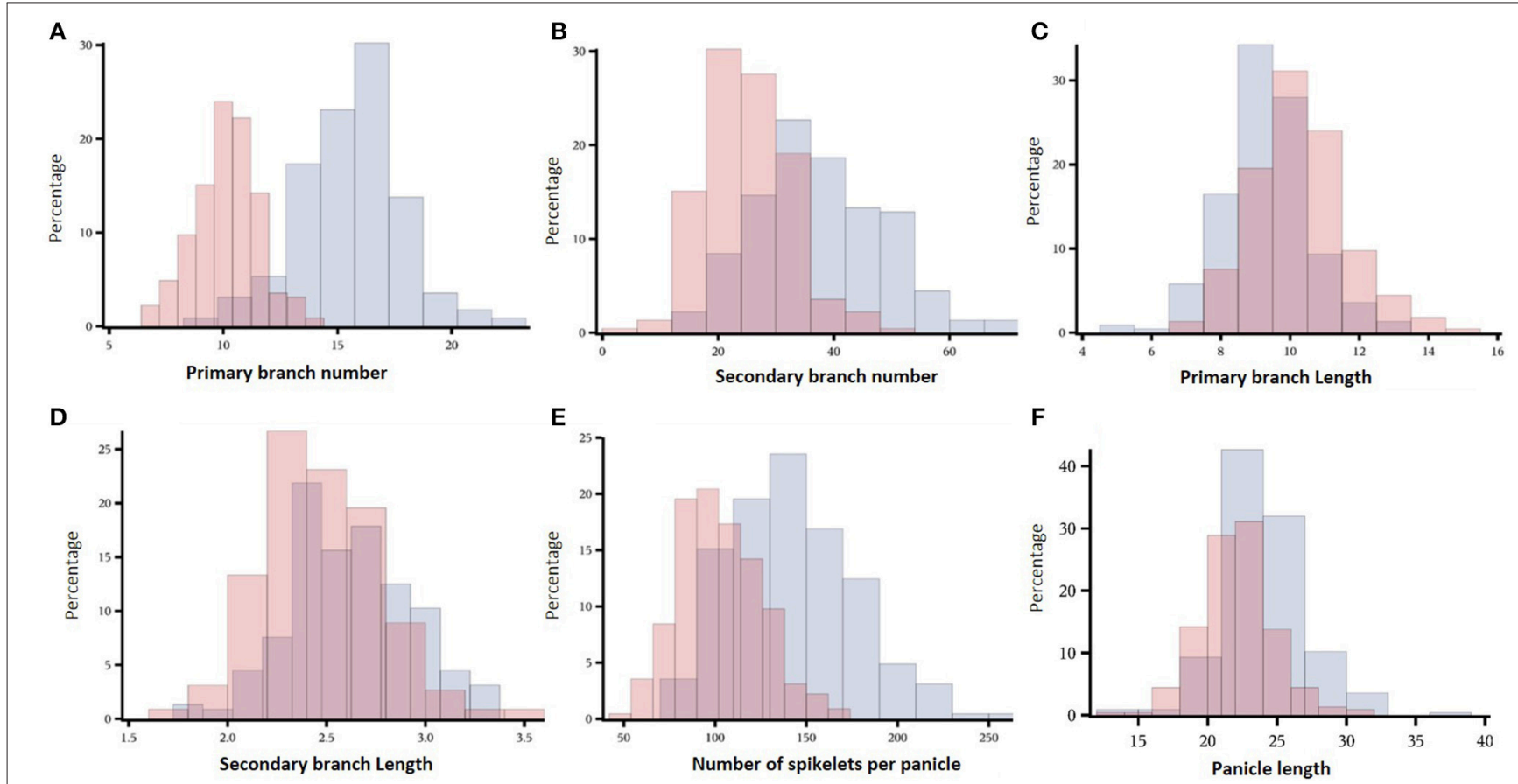

FIGURE 1 | Histograms of the distribution for panicle architecture related traits in both experiments. In blue and red are values for Exp1-TR and Exp2-DRS respectively. The percentage of individuals for each class is presented in the y-axis. (A) Primary branch number, (B) Secondary branch number, (C) Primary branch length, (D) Secondary branch length, (E) Number of spikelets per panicle, (F) Panicle length.

panicle architecture variables only in the cases of SBN in Exp2DRS (q-9) and PBN in Exp1-TR (q-43) (Figure 3). No collocation was observed between NSP and PBL or SBL within the same experiment. Finally, no GWAS collocations occurred between the number of branches (SBN, PBN) and the length of branches (SBL, $\mathrm{PBL}$ ) (Figure 3).

\section{Trait, Markers and Known Genes Associations}

A total of 21 GWAS sites were located near known genes that have been previously identified using mutants or studies of recombinant populations (Table 4).

Six GWAS sites associated with panicle architecture and the number of spikelets per panicle were located within the LD distance of 6 genes related to floral organ formation (Table 4). NECKLEAF1 (nl1) a gene controlling floral organ identity and flowering time was located within the LD block of q-25 associated with NSPexp1. Osmads6-5 and Lax genes affecting the number of spikelets per panicle and the number of branches were within the LD block of $\mathrm{q}-12$ and $\mathrm{q}-7$ respectively and associated with SBLexp1. Osmads5 was within the LD region of q-27 associated to SBNexp1, OsJAG gene which has a known function on the development of the panicle and the number of spikelets per panicle was near q-1 in chromosome 1 position 1.626.152 bp and associated with PBL in Exp2. Finally, Jmj6 controlling the number and morphology of reproductive organs was within the LD region of q-44 associated with PBNexp1. One GWAS site q-14 was near a gene controlling flowering time, 
TABLE 3 | Correlation between panicle architecture and number of spikelets per panicle for both experiments.

\begin{tabular}{|c|c|c|c|c|c|c|c|c|c|c|c|c|}
\hline & NSPexp1 & NSPexp2 & PBLexp1 & PBLexp2 & PBNexp1 & PBNexp2 & SBLexp1 & SBLexp2 & SBNexp1 & SBNexp2 & PLexp1 & PLexp2 \\
\hline NSPexp1 & 1 & & & & & & & & & & & \\
\hline NSPexp2 & $0.682^{\star *}$ & 1 & & & & & & & & & & \\
\hline PBLexp1 & $0.184^{\star}$ & 0.126 & 1 & & & & & & & & & \\
\hline PBLexp2 & 0.138 & $0.167^{\star}$ & $0.473^{\star *}$ & 1 & & & & & & & & \\
\hline PBNexp1 & $0.545^{\star \star}$ & $0.527^{\star *}$ & 0.058 & 0.119 & 1 & & & & & & & \\
\hline SBLexp2 & 0.079 & 0.035 & $0.406^{\star \star}$ & $0.770^{\star \star}$ & -0.015 & $-0.244^{\star}$ & $0.619^{\star *}$ & 1 & & & & \\
\hline SBNexp1 & $0.598^{\star \star}$ & $0.559^{\star}$ & $0.365^{\star *}$ & 0.118 & $0.719^{\star *}$ & $0.383^{\star *}$ & 0.145 & 0.002 & 1 & & & \\
\hline SBNexp2 & $0.589^{\star *}$ & $0.607^{\star}$ & -0.029 & $0.354^{\star *}$ & $0.483^{\star \star}$ & $0.541^{\star *}$ & -0.101 & 0.107 & $0.580^{\star *}$ & 1 & & \\
\hline PLexp1 & 0.094 & 0.099 & $0.635^{\star *}$ & $0.435^{\star \star}$ & $0.338^{\star *}$ & -0.044 & $0.421^{\star \star \star}$ & $0.307^{\star \star \star}$ & $0.398^{\star \star \star}$ & 0.095 & 1 & \\
\hline PLexp2 & 0.141 & $0.180^{\star}$ & $0.427^{\star \star}$ & $0.538^{\star \star}$ & 0.074 & $0.276^{\star \star}$ & $0.226^{\star}$ & $0.349^{\star *}$ & 0.161 & $0.350^{\star \star}$ & $0.400^{\star \star}$ & 1 \\
\hline
\end{tabular}

Values with ${ }^{* * *},{ }^{* *}$, and ${ }^{*}$ indicate correlations with $p<0.0001, p<0.01$, and $p<0.05$, respectively. Bold values indicate significant correlations between traits.

SBLexp1 in chromosome 3 was within the LD block of the gene OSMADS50.

Three GWAS sites q-6, q-23, q-26, q-28 associated with the number of spikelets per panicle or the number of primary branches were within the region of genes controlling plant nutrient status (ammonium, Iron, nitrogen content). Genes controlling plant size were within the LD region of GWAS sites associated with NSP or with SBL (q-22, q-15, q16, q23). The sites $\mathrm{q}-2, \mathrm{q}-3, \mathrm{q}-17, \mathrm{q}-20, \mathrm{q}-36, \mathrm{q}-37$ were within the LD regions of genes controlling grain size, pollen development or seed starch content suggesting a linkage between the genetic control of panicle architecture and grain formation.

Therefore, for all the panicle traits dissected with image analysis (PBL, PBN, SBL, and $\mathrm{SBN}$ ), we found significant associations at sites where known genes with relevant function were reported.

\section{GWAS Sites Detected across Experiments}

Thirty one GWAS sites were associated with variables measured in Exp1-TR and 17 GWAS sites were associated to variables measured in Exp2-DRS. Only 2 GWAS sites, namely q-13 and q-46 were detected in both experiments (Figure 3), indicating stability across contrasting conditions (direct seeding and transplanting), although no gene with known relevant function to the trait reported in these regions (Table 4).

Within the LD block of site q-46 in chromosome 11 associated with SBN in Exp2-DRS and NSP in Exp1-TR, we detected four SNPs at positions 23,012,580, 23,012,600, 23,047,814, and $23,064,262$ bp having significant associations with both trait and both environments. Within the indica panel, 155 accessions carrying the combination of AGAC at these markers showed superior values for NSP and SBN in both experiments (Figures 4A,B).

For site q-13 in chromosome 3 associated with SBLin Exp2DRS and SBL in Exp1-TR, four markers at the positions 519,551, $519,745,519,765$, and 550,877 bp were found, whereby 41 accessions carrying the combination of GGTT had significantly superior values for SBL in both experiments (Figure 4D).
However, these 41 accessions did not show higher values of NSP either in Exp1-TR or in Exp2-DRS (Figure 4C).

\section{DISCUSSION}

Despite some existing knowledge on the genetic control of the number of spikelets per panicle, there is little evidence on the added value for breeding of secondary traits, such as panicle architecture traits. This may be explained by the lack of undestanding of (i) the role of each panicle architecture component trait and their mutual compensations, (ii) the genetic architecture of panicle component traits, and (iii) the stability of the panicle component traits across contrasting environmental conditions.

The present study combined image analysis, GWAS and field trials under contrasting management conditions (transplanting vs. direct seeding) for the phenotypic and genetic dissection of the number of spikelets per panicle in rice. It was expected that the phenotyping of panicle architecture traits would provide further insight into trait-trait correlations and trade-offs defining the total number of spikelets per panicle, while the analysis of two contrasting conditions would provide insights into environmental and genetic control.

\section{Image Analysis of Panicle Architecture Enabled Phenotypic Dissection of Number of Spikelets per Panicle}

New open access image analysis tools such as the one used in this study (P-TRAP: AL-Tam et al., 2013) allowed us to evaluate the phenotypic contribution of panicle architecture to the number of spikelets per panicle in a large diversity panel. We observed a large range of variation and high heritability (Table 2) for the panicle architecture traits and the number of spikelets per panicle, suggesting a good performance of P-TRAP as a phenotyping tool. Crowell et al. (2014) using a different image analysis tool to dissect panicle architecture, also showed 


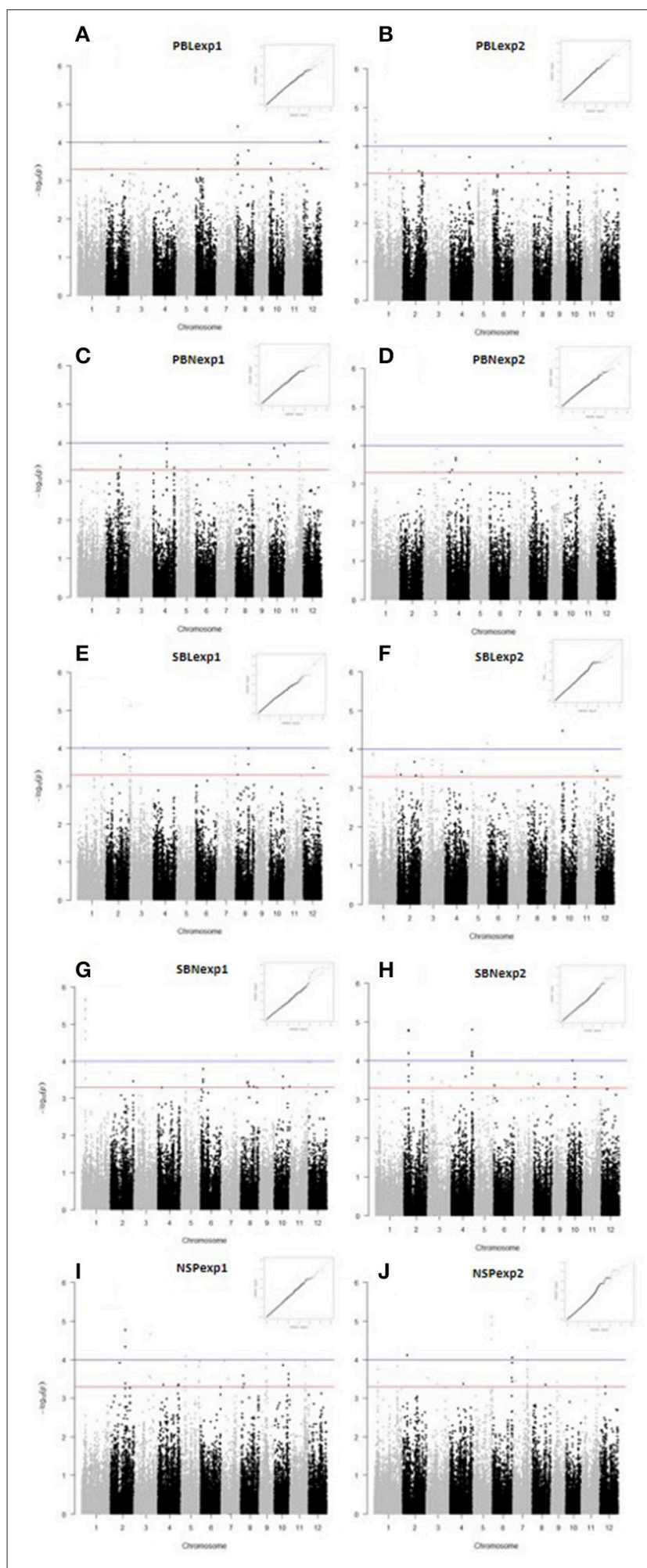

FIGURE 2 | Manhattan plots and Q-Q plots for panicle architecture related traits and number of spikelets per panicle. (A) PBLexp1, (B) PBLexp2, (C) PBNexp1, (D) PBNexp2, (E) SBLexp1, (F) SBLexp2, (G) SBNexp1, (H) SBNexp2, (I) NSPexp1, and (J) NSPexp2. In the Manhattan plots the blue line and red lines indicates the threshold for significant SNP association at $p<1 \times 10^{-4}$ and $p<5 \times 10^{-4}$ respectively. the advantage of image analysis over manual measurements to phenotype diversity panels.

Previous studies addressing panicle architecture highlighted the importance of each trait/gene according to its effect on the number of spikelets per panicle (Ikeda et al., 2004; Xu et al., 2004; Ando et al., 2008; Qiao et al., 2011; Hashida et al., 2013). In this study, NSP was significantly correlated with panicle architecture variables, confirming the positive effect of the number of branches (PBN, SBN) (Li et al., 2003; Wang et al., 2009) and branch length (PBL) (Xu et al., 2004; Li et al., 2009).

However, unlike in the aforementioned studies P-TRAP introduced new variables such as the number (SBN) and length (SBL) of secondary branches. Previous studies reported a positive correlation between SBN and NSP (Li et al., 2003; Wang et al., 2009). Hashida et al. (2013) observed an increased number of spikelets per panicle and secondary branches in an indica $\times$ japonica cross carrying the gene OsSPS1. Furthermore, several authors confirmed the importance of SBN over PBN as contributors to NSP in rice (Xu et al., 2004; Mei et al., 2006; Terao et al., 2010). To our knowledge studies addressing SBL are rare, mainly because of the difficulty to measure it manually and only Piao et al. (2009) showed that both SBL and SBN together contribute positively to the NSP. However, in this study, we did not observe a significant correlation between NSP and SBL (Table 3), in any of the growing conditions.

Variables related to the number of branches (PBN, SBN) were not significantly correlated with variables related to the length of branches (PBL), suggesting two independent ways to increase the NSP, either with a high number of branches or with long branches, involving trade-offs between them. The number of spikelets per panicle can be increased; either by increasing the number of branches (Xu et al., 2004; Wang et al., 2009; Qi et al., 2011) or by increasing the length of branches ( $\mathrm{Li}$ et al., 2009). In fact, none of the GWAS sites associated with variables related to number and length of branches collocated, suggesting that the number and length of primary branches have an independent genetic control. To our knowledge, no study has addressed both length and number of branches to increase the number of spikelets per panicle. This may open a new way to increase NSP as suggested by Ando et al. (2008).

PL was significantly and positive correlated with NSP in Exp2-DRS and no correlation was found between PL and NSP in Exp1-TR. However, all panicle component traits (SBN, SBL, $\mathrm{PBN}$, and $\mathrm{PBL}$ ) were significantly and positively correlated with PL under both environmental conditions. This suggest that PL is not a simple trait and can be affected in a given genotype, by several component traits (SBN, SBL, PBN, or/and $\mathrm{PBL}$ ) that are physiologically interacting and may involve environment dependent trade-offs. Thus, the low correlation observed between PL and NSP in this study can be explained by the effect of the growing conditions on PL and/or the different ways a genotype can achieve a large number of spikelets per panicle (either with long panicles or with short but dense panicles). 


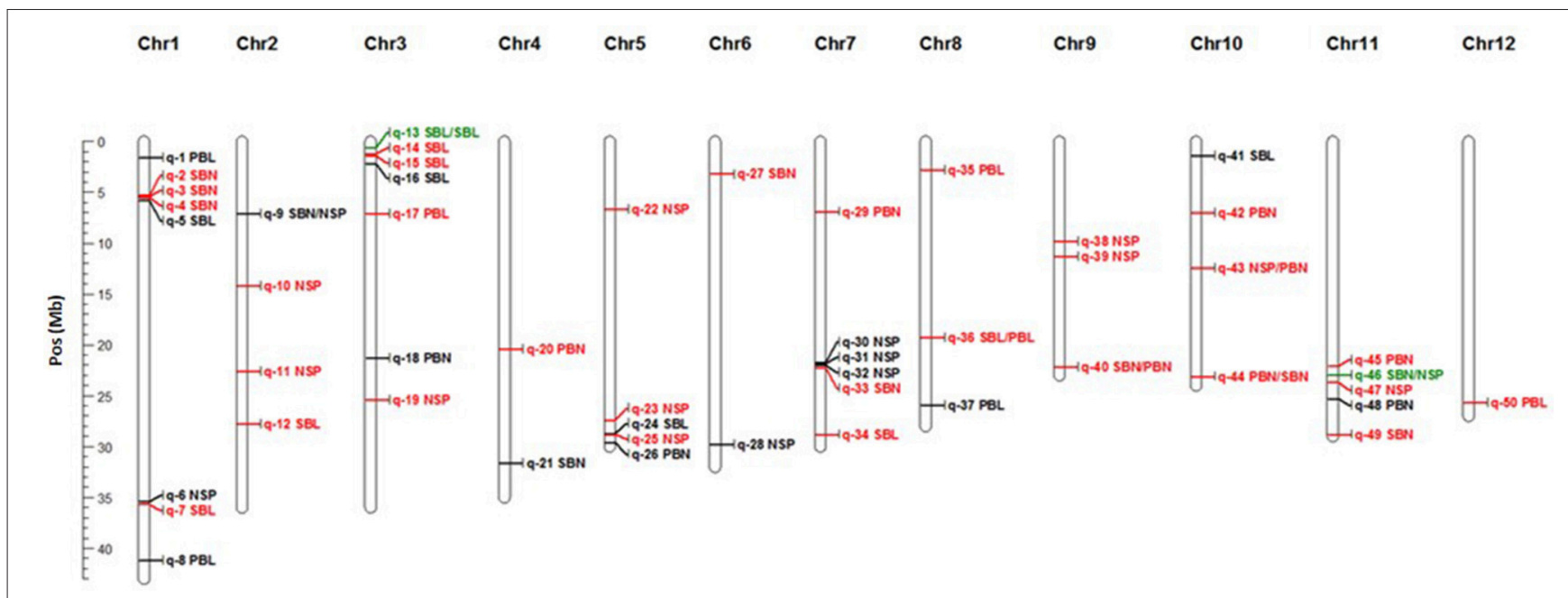

FIGURE 3 | Physical map position of significant GWAS sites detected through MLM1. In red GWAS sites for Exp1-TR, in black GWAS site for Exp2-DRS and in green GWAS sites across experiments. Co-locations are represented by "/" between traits.

\section{GWAS on Panicle Architecture Dissects Genetic Control of Number of Spikelets per Panicle}

The exploration of a diversity panel using GWAS and image analysis of panicle architecture allowed us to find 25 new loci that were involved in the genotypic variation of NSP a complex trait (Table 3). A total of 23 GWAS sites had significant associations $\left(p<1.00 \mathrm{E}^{-4}\right)$ with panicle architecture traits. Furthermore, SNPs with high association probabilities were more likely to be close to previously identified candidate genes, e.g., Osjag in q-1 and $O s C K X 2$ in $\mathrm{q}-3$ with $\mathrm{p}$ values of $7.70 \mathrm{E}-5$ and $1.54 \mathrm{E}-5$ respectively. Besides, image analysis allowed us to measure new variables, such as SBN, that were related with the number of spikelets per panicle and associated with new GWAS sites, some of which related with known genes (OsCKX2, OsMADS5). Thus, this GWAS study identified many true genotype-phenotype associations, and detected GWAS sites that were associated to already known genes with a highly significant effect on the number of spikelets per panicle (Table 4).

Ten GWAS sites associated only with NSP remain interesting markers that might be associated with a component trait of NSP that was not measured in this study (Qiao et al., 2011; Hashida et al., 2013).

Numerous markers with significant but small effect were also found. This was also the case in other association studies for panicle architecture traits in rice (Crowell et al., 2016) and sorghum (Morris et al., 2013; Hmon et al., 2014), confirming that panicle architecture is controlled by many QTLs with small effect. So far, several GWAS sites having significant associations at $P<$ $5.5 \mathrm{E}^{-4}$ and associated with genes controlling panicle architecture have been identified and characterized independently. The genes such as MOC1, LAX1/2, OsCKX2, SP1, DEP1/2/3, and IPA1/WFP were found to modify panicle architecture and were suggested as candidates to improve the number of spikelets per panicle in rice through molecular breeding (Komatsu K. et al., 2003; Ashikari et al., 2005; Miura et al., 2010; Qiao et al., 2011). Interestingly, Wang and Li (2011) and Liang et al. (2014) suggested that their effect on the final number of spikelets per panicle would be greater if they were all included in the breeding design, as they all act in an additive way.

Co-localization of genetic control of various traits in the same genomic region was rare in this study (7 out of 50 GWAS sites) compared with other studies (Rebolledo et al., 2015). Considering the $\mathrm{LD}$ region of $75 \mathrm{~kb}$, we found two co-localizations between panicle architecture traits and NSP (q-9, q-43) suggesting that component traits derived from the image analysis of panicle architecture strengthened the analysis of NSP.

Globally, in our study only 14 GWAS sites out of 50 were significantly associated with NSP only. Four out of the 14 GWAS sites for NSP were related to known, cloned genes; two GWAS sites were associated with genes with a function affecting panicle density (q-22, gene GSK22) or floral organ identity (q25 , gene NL1) and two genes with a function on tillering ability (q-23, OsHRZ2 gene) or carbon and nitrogen content ( $\mathrm{q}-6$, gene OSAAT7).

\section{Contrasting Field Trials Revealed Specific and Common GWAS Sites}

The Exp2-DRS showed lower values for NSP, PBN, SBN than Exp1-TR across the 225 varieties (Table 2). The negative effect of direct seeding on the number of spikelets per panicle was reported by Huang et al. (2011) for super hybrid rice, showing that a smaller amount of nutrients available under direct seeding at panicle initiation affected panicle branching and the final number of spikelets per panicle. This result reflects the high degree of sensitivity to the environment of panicle architecture, making the detection of genetic controls more difficult.

However, we detected two GWAS sites consistently in both transplanting and direct seeding conditions. Site q-13 was stable 


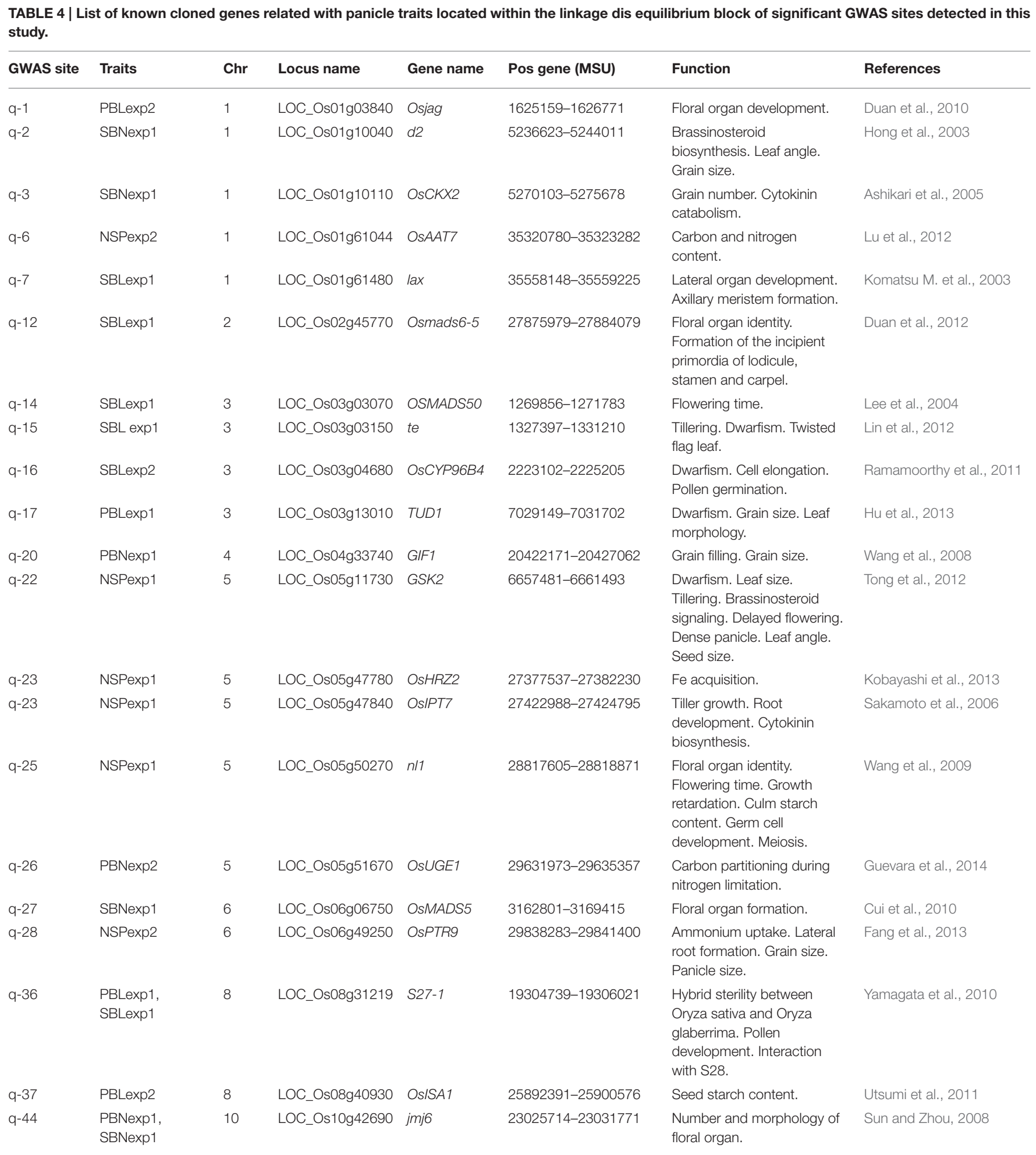

across conditions and was associated with branching pattern (SBL) but did not have a significant effect on NSP. This result supports the idea that the length of the branches has a smaller effect on the number of spikelets per panicle (Ando et al., 2008).
Site q-46 was also stable in Exp1-TR and Exp2-DRS and controlled both SBN and NSP. Thus, new variables measured in this study using image analysis led to the discovery of new genetic regions with a significant effect on NSP across two different rice growing conditions. 

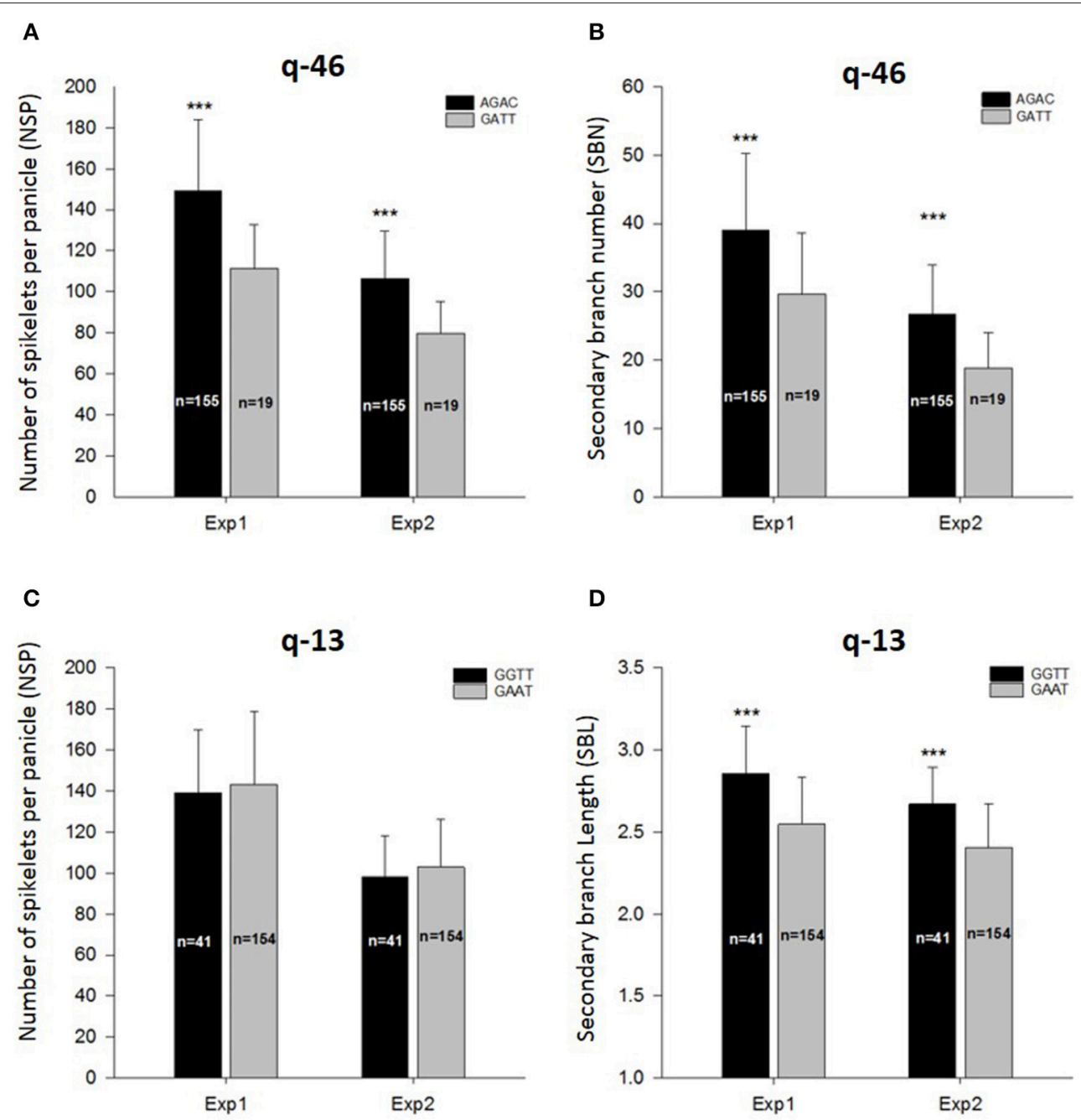

D

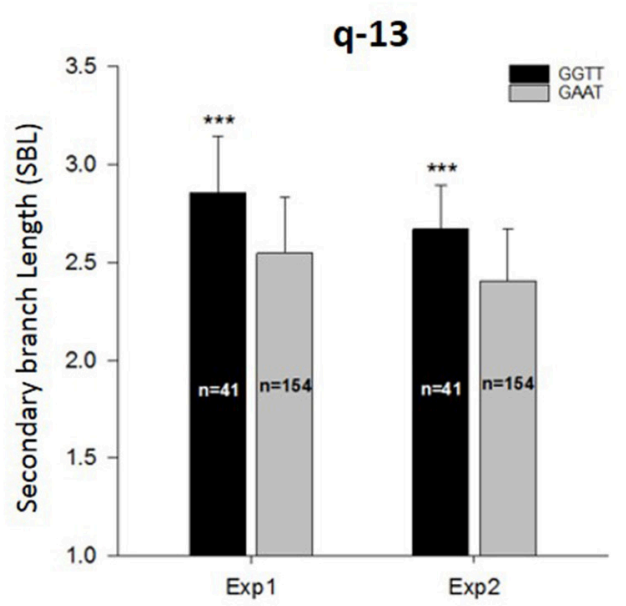

FIGURE 4 | Allelic combination for q-46 (A,B) and q-13 (C,D), and their effect on the number of spikelets per panicle, secondary branch number and secondary branch length. ***Indicate significant differences at $p<0.001$ between allelic combination for each experiment.

Hence, despite the strong environment effect, some genomic regions associated with multiple traits point to the potential for improvement of yield potential through increased number of spikelets per panicle for both transplanting and direct seeding conditions. But overall, it appears that molecular breeding for increased NSP can build on more relevant loci when targeting a specific environmental and cultural practice.

\section{AUTHOR CONTRIBUTIONS}

MR designed the project, MR and AP analyzed and interpreted the data, also they wrote the manuscript. MD led the GRiSP Global Rice Phenotyping Network which this study was part of. All authors made important intellectual contributions. JD and DC performed the bioinformatic analysis of the GBS data.

\section{ACKNOWLEDGMENTS}

We thank Dr. JMC Pasuquin, Manager of the GRiSP Global Rice Phenotyping Network at IRRI, for valuable technical assistance and data provision; and the Global Rice Science Partnership for partial funding of this study through the PRAY New Frontier Project. D. Gonzalez, P. Sepulveda, E. Petro for their work gathering the data. Helene Adam for the advise on the experimental setup and Image Analysis using PTRAP.

\section{SUPPLEMENTARY MATERIAL}

The Supplementary Material for this article can be found online at: http://journal.frontiersin.org/article/10.3389/fpls.2016. 01384 


\section{REFERENCES}

AL-Tam, F., Adam, H., dos Anjos, A., Lorieux, M., Larmande, P., Ghesquière, A., et al. (2013). P-TRAP: a panicle trait phenotyping tool. BMC Plant Biol. 13:122. doi: 10.1186/1471-2229-13-122

Ando, T., Yamamoto, T., Shimizu, T., Ma, X. F., Shomura, A., Takeuchi, Y., et al. (2008). Genetic dissection and pyramiding of quantitative traits for panicle architecture by using chromosomal segment substitution lines in rice. Theor. Appl. Genet. 116, 881-890. doi: 10.1007/s00122-008-0722-6

Ashikari, M., Sakakibara, H., Lin, S., Yamamoto, T., Takashi, T., Nishimura, A., et al. (2005). Cytokinin oxidase regulates rice grain production. Science 309, 741-745. doi: 10.1126/science.1113373

Crowell, S., Falcão, A. X., Shah, A., Wilson, Z., Greenberg, A. J., and McCouch, S. R. (2014). High-resolution inflorescence phenotyping using a novel image-analysis pipeline, PANorama. Plant Physiol. 165, 479-495. doi: $10.1104 /$ pp.114.238626

Crowell, S., Korniliev, P., Falcão, A., Ismail, A., Gregorio, G., Mezey, J., et al. (2016). Genome-wide association and high-resolution phenotyping link Oryza sativa panicle traits to numerous trait-specific QTL clusters. Nat. Commun. 7:10527. doi: 10.1038/ncomms10527

Cui, R., Han, J., Zhao, S., Su, K., Wu, F., Du, X., et al. (2010). Functional conservation and diversification of class $\mathrm{E}$ floral homeotic genes in rice (Oryza sativa). Plant J. 61, 767-781. doi: 10.1111/j.1365-313X.2009.04101.x

Dingkuhn, M., Laza, M. R. C., Kumar, U., Mendez, K. S., Collard, B., Jagadish, K. S. V., et al. (2015). Improving yield potential of tropical rice: achieved levels and perspectives through improved ideotypes. Field Crops Res. 182, 43-59. doi: 10.1016/j.fcr.2015.05.025

Dingkuhn, M., Penning de Vries, F. W. T., De Datta, S. K., and van Laar, H. H. (1991). Concepts for a New Plant type for Direct Seeded Flooded Tropical Rice. Manila, Philippines: International Rice Research Institute.

Dixit, S., Grondin, A., Lee, C.-R., Henry, A., Olds, T.-M., and Kumar, A. (2015). Understanding rice adaptation to varying agro-ecosystems: trait interactions and quantitative trait loci. BMC Genet. 16:86. doi: 10.1186/s12863-0150249-1

Duan, Y., Diao, Z., Liu, H., Cai, M., Wang, F., Lan, T., et al. (2010). Molecular cloning and functional characterization of OsJAG gene based on a completedeletion mutant in rice (Oryza sativa L.). Plant Mol. Biol. 74, 605-615. doi: 10.1007/s11103-010-9703-7

Duan, Y., Xing, Z., Diao, Z., Xu, W., Li, S., Du, X., et al. (2012). Characterization of Osmads6-5, a null allele, reveals that OsMADS6 is a critical regulator for early flower development in rice (Oryza sativa L.). Plant Mol. Biol. 80, 429-442. doi: 10.1007/s11103-012-9958-2

Duitama, J., Quintero, J. C., Cruz, D. F., Quintero, C., Hubmann, G., FoulquiéMoreno, M. R., et al. (2014). An integrated framework for discovery and genotyping of genomic variants from high-throughput sequencing experiments. Nucleic Acids Res. 42:e44. doi: 10.1093/nar/gkt1381

Elshire, R. J., Glaubitz, J. C., Sun, Q., Poland, J. A., Kawamoto, K., Buckler, E. S., et al. (2011). A robust, simple genotyping-by-sequencing (GBS) approach for high diversity species. PLoS ONE 6:e19379. doi: 10.1371/journal.pone.0019379

Fang, Z., Xia, K., Yang, X., Grotemeyer, M. S., Meier, S., Rentsch, D., et al. (2013). Altered expression of the PTR/NRT1 homologue OsPTR9 affects nitrogen utilization efficiency, growth and grain yield in rice. Plant Biotechnol. J. 11, 446-458. doi: 10.1111/pbi.12031

Farooq, M., Siddique, K. H. M., Rehman, H., Aziz, T., Lee, D.-J., and Wahid, A. (2011). Rice direct seeding: experiences, challenges and opportunities. Soil Tillage Res. 111, 87-98. doi: 10.1016/j.still.2010.10.008

Foulkes, M. J., Slafer, G. A., Davies, W. J., Berry, P. M., Sylvester-Bradley, R., Martre, P., et al. (2011). Raising yield potential of wheat. III. Optimizing partitioning to grain while maintaining lodging resistance. J. Exp. Bot. 62, 469-486. doi: 10.1093/jxb/erq300

Fujita, D., Trijatmiko, K. R., Tagle, A. G., Sapasap, M. V., Koide, Y., Sasaki, K., et al. (2013). NAL1 allele from a rice landrace greatly increases yield in modern indica cultivars. Proc. Natl. Acad. Sci. U.S.A. 110, 20431-20436. doi: $10.1073 /$ pnas. 1310790110

Guevara, D. R., El-Kereamy, A., Yaish, M. W., Mei-Bi, Y., and Rothstein, S. J. (2014). Functional characterization of the rice UDP-glucose 4-epimerase 1, OsUGE1: a potential role in cell wall carbohydrate partitioning during limiting nitrogen conditions. PLoS ONE 9:e96158. doi: 10.1371/journal.pone.0096158
Hashida, Y., Aoki, N., Kawanishi, H., Okamura, M., Ebitani, T., Hirose, T., et al. (2013). A near isogenic line of rice carrying chromosome segments containing OsSPS1 of Kasalath in the genetic background of Koshihikari produces an increased spikelet number per panicle. Field Crops Res. 149, 56-62. doi: 10.1016/j.fcr.2013.04.020

Hmon, K. P. W., Shehzad, T., and Okuno, K. (2014). QTLs underlying inflorescence architecture in sorghum (Sorghum bicolor (L.) Moench) as detected by association analysis. Genet. Resour. Crop Evol. 61, 1545-1564. doi: 10.1007/s10722-014-0129-y

Hong, Z., Ueguchi-Tanaka, M., Umemura, K., Uozu, S., Fujioka, S., Takatsuto, S., et al. (2003). A rice brassinosteroid-deficient mutant, ebisu dwarf (d2), is caused by a loss of function of a new member of cytochrome P450. Plant Cell 15, 2900-2910. doi: 10.1105/tpc.014712

Huang, M., Zou, Y., Jiang, P., Xia, B., Feng, Y., Cheng, Z., et al. (2011). Yield component differences between direct-seeded and transplanted super hybrid rice. Plant Prod. Sci. 14, 331-338. doi: 10.1626/pps.14.331

Hu, X., Qian, Q., Xu, T., Zhang, Y. E., Dong, G., Gao, T., et al. (2013). The Ubox E3 ubiquitin ligase TUD1 functions with a heterotrimeric $\mathrm{G} \alpha$ subunit to regulate brassinosteroid-mediated growth in rice. PLoS Genet. 9:e1003391. doi: 10.1371/journal.pgen.1003391

Ikeda, K., Sunohara, H., and Nagato, Y. (2004). Developmental course of inflorescence and spikelet in rice. Breed. Sci. 54, 147-156. doi: 10.1270/jsbbs. 54.147

Kawahara, Y., de la Bastide, M., Hamilton, J. P., Kanamori, H., McCombie, W. R., Ouyang, S., et al. (2013). Improvement of the Oryza sativa Nipponbare reference genome using next generation sequence and optical map data. Rice 6:4. doi: 10.1186/1939-8433-6-4

Kobayashi, T., Nagasaka, S., Senoura, T., Itai, R. N., Nakanishi, H., and Nishizawa, N. K. (2013). Iron-binding haemerythrin RING ubiquitin ligases regulate plant iron responses and accumulation. Nat. Commun. 4:2792. doi: 10.1038/ncomm s3792

Komatsu, K., Maekawa, M., Ujiie, S., Satake, Y., Furutani, I., Okamoto, H., et al. (2003). LAX and SPA: major regulators of shoot branching in rice. Proc. Natil. Acad. Sci. U.S.A. 100, 11765-11770. doi: 10.1073/pnas.1932414100

Komatsu, M., Maekawa, M., Shimamoto, K., and Kyozuka, J. (2003). The LAX1 and FRIZZY PANICLE 2 genes determine the inflorescence architecture of rice by controlling rachis-branch and spikelet development. Dev. Biol. 231, 364-373. doi: 10.1006/dbio.2000.9988

Kumar, V., and Ladha, J. K. (2011). Direct seeding of rice. Adv. Agron. 111, 297-413. doi: 10.1016/B978-0-12-387689-8.00001-1

Laza, M. R. C., Sakai, H., Cheng, W., Tokida, T., Peng, S., and Hasegawa, T. (2015). Differential response of rice plants to high night temperatures imposed at varying developmental phases. Agric. Forest Meteorol. 209, 69-77. doi: 10.1016/j.agrformet.2015.04.029

Lee, S., Kim, J., Han, J. J., Han, M. J., and An, G. (2004). Functional analyses of the flowering time gene OsMADS50, the putative SUPPRESSOR OF OVEREXPRESSION OF CO 1/AGAMOUS-LIKE 20 (SOC1/AGL20) ortholog in rice. Plant J. 38, 754-764. doi: 10.1111/j.1365-313X.2004.02082.X

Liang, W. H., Shang, F., Lin, Q. T., Lou, C., and Zhang, J. (2014). Tillering and panicle branching genes in rice. Gene 537, 1-5. doi: 10.1016/j.gene.2013.11.058

Lin, Q., Wang, D., Dong, H., Gu, S., Cheng, Z., Gong, J., et al. (2012). Rice APC/CTE controls tillering by mediating the degradation of MONOCULM 1. Nat. Commun. 3:752. doi: 10.1038/ncomms1716

Li, S., Qian, Q., Fu, Z., Zeng, D., Meng, X., Kyozuka, J., et al. (2009). Short panicle1 encodes a putative PTR family transporter and determines rice panicle size. Plant J. 58, 592-605. doi: 10.1111/j.1365-313X.2009.03799.x

Li, X., Qian, Q., Fu, Z., Wang, Y., Xiong, G., Zeng, D., et al. (2003). Control of tillering in rice. Nature 422, 618-621. doi: 10.1038/nature01518

Li, X., Yan, W., Agrama, H., Jia, L., Jackson, A., Moldenhauer, K., et al. (2012). Unraveling the complex trait of harvest index with association mapping in rice (Oryza sativa L.). PLoS ONE 7:e29350. doi: 10.1371/journal.pone.0029350

Lu, Y., Song, Z., Lü, K., Lian, X., and Cai, H. (2012). Molecular characterization, expression and functional analysis of the amino acid transporter gene family (OsAATs) in rice. Acta Physiol. Plant. 34, 1943-1962. doi: 10.1007/s11738-0120995-X

Mather, K. A., Caicedo, A. L., Polato, N. R., Olsen, K. M., McCouch, S., and Purugganan, M. D. (2007). The extent of linkage disequilibrium in rice (Oryza sativa L.). Genetics 177, 2223-2232. doi: 10.1534/genetics.107.079616 
Mei, H. W., Xu, J. L., Li, Z. K., Yu, X. Q., Guo, L. B., Wang, Y. P. et al. (2006). QTLs influencing panicle size detected in two reciprocal introgressive line (IL) populations in rice (Oryza sativa L.). Theor. Appl. Genet. 112, 648-656. doi: 10.1007/s00122-005-0167-0

Miura, K., Ikeda, M., Matsubara, A., Song, X. J., Ito, M., Asano, K., et al. (2010). OsSPL14 promotes panicle branching and higher grain productivity in rice. Nat. Genet. 42, 545-549. doi: 10.1038/ng.592

Morris, G. P., Ramu, P., Deshpande, S. P., Hash, C. T., Shah, T., Upadhyaya, H. D., et al. (2013). Population genomic and genome-wide association studies of agroclimatic traits in sorghum. Proc. Natl. Acad. Sci. U.S.A. 110, 453-458. doi: 10.1073/pnas.1215985110

Norton, G. J., Douglas, A., Lahner, B., Yakubova, E., Guerinot, M. L., Pinson, S. R., et al. (2014). Genome wide association mapping of grain arsenic, copper, molybdenum and zinc in rice (Oryza sativa L.) grown at four international field sites. PLoS ONE 9:e89685. doi: 10.1371/journal.pone.0089685

Pantuwan, G., Fukai, S., Cooper, M., Rajatasereekul, S., and O'toole, J. C. (2002). Yield response of rice (Oryza sativa L.) genotypes to drought under rainfed lowlands: 2. Selection of drought resistant genotypes. Field Crops Res. 73, 169-180. doi: 10.1016/S0378-4290(01)00195-2

Peng, S., Cassman, K. G., Virmani, S. S., Sheehy, J., and Khush, G. S. (1999). Yield potential trends of tropical rice since the release of IR8 and the challenge of increasing rice yield potential. Crop Sci. 39, 1552-1559. doi: 10.2135/cropsci1999.3961552x

Peng, Y., Gao, Z., Zhang, B., Liu, C., Xu, J., Ruan, B., et al. (2014). Fine mapping and candidate gene analysis of a major QTL for panicle structure in rice. Plant Cell Rep. 33, 1843-1850. doi: 10.1007/s00299-014-1661-0

Perea, C., Hoz, J. F. D. L., Cruz, D. F., Lobaton, J. D., Izquierdo, P., Quintero, J. C., et al. (2016). Bioinformatic analysis of genotype by sequencing (GBS) data with NGSEP. BMC Genomics. 17:498. doi: 10.1186/s12864-016-2827-7

Piao, R., Jiang, W., Ham, T. H., Choi, M. S., Qiao, Y., Chu, S. H., et al. (2009). Mapbased cloning of the ERECT PANICLE 3 gene in rice. Theor. Appl. Genet. 119, 1497-1506. doi: 10.1007/s00122-009-1151-x

Qi, W., Sun, F., Wang, Q., Chen, M., Huang, Y., Feng, Y. Q., et al. (2011). Rice ethylene-response AP2/ERF factor OsEATB restricts internode elongation by down-regulating a gibberellin biosynthetic gene. Plant Physiol. 157, 216-228. doi: 10.1104/pp.111.179945

Qiao, Y., Piao, R., Shi, J., Lee, S. I., Jiang, W., Kim, B. K., et al. (2011). Fine mapping and candidate gene analysis of dense and erect panicle 3, DEP3, which confers high grain yield in rice (Oryza sativa L.). Theor. Appl. Genet. 122, 1439-1449. doi: 10.1007/s00122-011-1543-6

Ramamoorthy, R., Jiang, S. Y., and Ramachandran, S. (2011). Oryza sativa cytochrome $\mathrm{P} 450$ family member OsCYP96B4 reduces plant height in a transcript dosage dependent manner. PLoS ONE 6:e28069. doi: 10.1371/journal.pone.0028069

Ray, D. K., Mueller, N. D., West, P. C., and Foley, J. A. (2013). Yield trends are insufficient to double global crop production by 2050. PLoS ONE 8:e66428. doi: 10.1371/journal.pone.0066428

Rebolledo, M. C., Dingkuhn, M., Courtois, B., Gibon, Y., Clément-Vidal, A., Cruz, D. F., et al. (2015). Phenotypic and genetic dissection of component traits for early vigour in rice using plant growth modelling, sugar content analyses and association mapping. J. Exp. Bot. 66, 5555-5566. doi: 10.1093/jxb/erv258

Sakamoto, T., Sakakibara, H., Kojima, M., Yamamoto, Y., Nagasaki, H., Inukai, Y., et al. (2006). Ectopic expression of KNOTTED1-like homeobox protein induces expression of cytokinin biosynthesis genes in rice. Plant Physiol. 142, 54-62. doi: 10.1104/pp.106.085811

Scheet, P., and Stephens, M. (2006). A fast and flexible statistical model for largescale population genotype data: applications to inferring missing genotypes and haplotypic phase. Am. J. Hum. Genet. 78, 629-644. doi: 10.1086/502802
Sreenivasulu, N., and Schnurbusch, T. (2012). A genetic playground for enhancing grain number in cereals. Trends Plant Sci. 17, 91-101. doi: 10.1016/j.tplants.2011.11.003

Sun, Q., and Zhou, D. X. (2008). Rice jmjC domain-containing gene JMJ706 encodes H3K9 demethylase required for floral organ development. Proc. Natl. Acad. Sci. U.S.A. 105, 13679-13684. doi: 10.1073/pnas.0805901105

Terao, T., Nagata, K., Morino, K., Hirose, T. (2010). A gene controlling the number of primary rachis branches also controls the vascular bundle formation and hence is responsible to increase the harvest index and grain yield in rice. Theor. Appl. Genet. 120, 875-893. doi: 10.1007/s00122-009-1218-8

The 3000 rice genomes project (2014). The 3,000 rice genomes project. GigaScience 3:7. doi: 10.1186/2047-217X-3-7

Tong, H., Liu, L., Jin, Y., Du, L., Yin, Y., Qian, Q., et al. (2012). DWARF AND LOW-TILLERING acts as a direct downstream target of a GSK3/SHAGGY-like kinase to mediate brassinosteroid responses in rice. Plant Cell 24, 2562-2577. doi: 10.1105/tpc.112.097394

Ueda, Y., Frimpong, F., Qi, Y., Matthus, E., Wu, L., Höller, S., et al. (2015). Genetic dissection of ozone tolerance in rice (Oryza sativa L.) by a genome-wide association study. J. Exp. Bot. 66, 293-306. doi: 10.1093/jxb/eru419

Utsumi, Y., Utsumi, C., Sawada, T., Fujita, N., and Nakamura, Y. (2011). Functional diversity of isoamylase oligomers: the ISA1 homo-oligomer is essential for amylopectin biosynthesis in rice endosperm. Plant Physiol. 156, 61-77. doi: 10.1104/pp.111.173435

Wade, L. J., Bartolome, V., Mauleon, R., Vasant, V. D., Prabakar, S. M., Chelliah, M., et al. (2015). Environmental response and genomic regions correlated with rice root growth and yield under drought in the OryzaSNP panel across multiple study systems. PLoS ONE 10:e0124127. doi: 10.1371/journal.pone.0124127

Wang, E., Wang, J., Zhu, X., Hao, W., Wang, L., Li, Q., et al. (2008). Control of rice grain-filling and yield by a gene with a potential signature of domestication. Nat. Genet. 40, 1370-1374. doi: 10.1038/ng.220

Wang, L., Yin, H., Qian, Q., Yang, J., Huang, C., Hu, X., et al. (2009). NECK LEAF 1, a GATA type transcription factor, modulates organogenesis by regulating the expression of multiple regulatory genes during reproductive development in rice. Cell Res. 19, 598-611. doi: 10.1038/cr.2009.36

Wang, Y., and Li, J. (2011). Branching in rice. Curr. Opin. Plant Biol. 14, 94-99. doi: 10.1016/j.pbi.2010.11.002

Xu, J. L., Yu, S. B., Luo, L. J., Zhong, D. B., Mei, H. W., and Li, Z. K. (2004). Molecular dissection of the primary sink size and its related traits in rice. Plant Breed. 123, 43-50. doi: 10.1046/j.1439-0523.2003.00936.x

Yamagata, Y., Yamamoto, E., Aya, K., Win, K. T., Doi, K., Ito, T., et al. (2010). Mitochondrial gene in the nuclear genome induces reproductive barrier in rice. Proc. Natl. Acad. Sci. U.S.A. 107, 1494-1499. doi: 10.1073/pnas.0908283107

Zhuang, J. Y., Lin, H. X., Lu, J., Qian, H. R., Hittalmani, S., Huang, N., et al. (1997). Analysis of QTL $\times$ environment interaction for yield components and plant height in rice. Theor. Appl. Genet. 95, 799-808. doi: 10.1007/s001220050628

Conflict of Interest Statement: The authors declare that the research was conducted in the absence of any commercial or financial relationships that could be construed as a potential conflict of interest.

Copyright $\odot 2016$ Rebolledo, Peña, Duitama, Cruz, Dingkuhn, Grenier and Tohme. This is an open-access article distributed under the terms of the Creative Commons Attribution License (CC BY). The use, distribution or reproduction in other forums is permitted, provided the original author(s) or licensor are credited and that the original publication in this journal is cited, in accordance with accepted academic practice. No use, distribution or reproduction is permitted which does not comply with these terms. 